\title{
Remote Programming of Cardiac Implantable Electronic Devices for MRI: Are We Ready to Change the Channel?
}

\author{
Ansel Amaral ${ }^{1}$ and Jay Montgomery ${ }^{1}$ \\ ${ }^{1}$ Vanderbilt University Medical Center
}

February 28, 2022

\begin{abstract}
Other editorials do not seem to include editorials. If you are sure you want one, let me know.
\end{abstract}

Editorial: Remote Programming of Cardiac Implantable Electronic Devices for MRI: Are We Ready to Change the Channel?

Authors:

Ansel Philip Amaral, MD, PhD

Jay Alan Montgomery, MD

Affiliation:

Division of Cardiovascular Medicine, Vanderbilt University Medical Center, Nashville, TN

Word Count: 1238

Number of Tables: 2

Funding: None

Disclosures: (None)

Magnetic resonance imaging (MRI) has become an indispensable diagnostic tool across many fields of clinical medicine. Although reprogramming of cardiac implantable electronic devices (CIEDs) for MRI is now routine at most institutions, early experiences were notable for potential adverse effects such as device-related heating, device or lead movement, and device malfunction. ${ }^{1}$ The American Society for Testing and Materials defines three categories of MRI safety (safe, conditional, and unsafe), as shown in Table $1 .^{2}$ Additionally, the term 'nonconditional' was included in the 2017 Heart Rhythm Society guidelines, which describes the up-to-date recommendations for CIEDs in MRI. ${ }^{3}$ To date, the Food and Drug Administration (FDA) has yet to declare a CIED as MR safe. ${ }^{2}$ The 2017 Heart Rhythm Society Guidelines make a class I recommendation for MRI with MR conditional systems in the context of a standardized institutional workflow and a class IIa recommendation for MR nonconditional systems in the absence of fractured, epicardial, or abandoned leads. ${ }^{3}$ Furthermore, the guidelines suggest a standardized institutional policy which includes a risk/benefit assessment of MRI compared to alternate imaging modalities, protocols for pre-scan and post-scan CIED evaluation, appropriate programming during the scan based on device and patient characteristics, and procedures in the event of an adverse clinical event. ${ }^{3}$

At our institution, the standardized protocol for any MRI includes a "checklist" that includes the presence or absence of a CIED. Once a CIED is identified, the system is identified as MR conditional or MR 
nonconditional with a concurrent effort to identify fractured, epicardial, or abandoned leads. If a clinical decision is made to proceed with MRI, pacing dependence is then determined. An asynchronous pacing mode (VOO/DOO) is chosen if the patient is pacing-dependent and sensing-only (OVO/ODO) mode is chosen if non-dependent. An inhibiting (VVI/DDI) mode can also be chosen if the patient is non-dependent. However, our preference has been to avoid this mode due to the risk of inappropriate sensing, which has been previously described. ${ }^{4}$ Asynchronous pacing rates are programmed faster than the underlying rate to avoid competitive pacing. Lastly, tachycardia detections and therapies are programmed off. The programming changes are prescribed by the Arrhythmia Section and typically performed by a field services representative from the individual CIED manufacturer. Qualified personnel trained in ACLS and an external defibrillator are readily available in the case of an adverse clinical event. At the completion of the MRI, the patient's CIED is checked for stability of device and lead parameters and subsequently reprogrammed to pre-MRI settings. Most institutions adhere to similar protocols, with a relatively low adverse event rate. ${ }^{1}$

In this issue of the Journal of Cardiovascular Electrophysiology, Siddamsetti et al. ${ }^{5}$ present a novel approach to programming CIEDs for MRI. The paper describes and analyzes a local protocol for remote reprogramming of MR conditional Medtronic (Minneapolis, MN, USA) CIEDs for MRI by a Medtronic field services representative. In this single-center, observational study conducted over a course of 20 months, 209 consecutive patients requiring an MRI underwent remote reprogramming of their CIED. Interestingly, the primary in-person procedural interaction at the time of reprogramming was performed by the on-site MRI technician with the Medtronic 2090 programmer while the programming was performed by an off-site field services representative from the device manufacturer. Remote control of the Medtronic 2090 programmer was established following access code verification. CIEDs were programmed to a pre-specified MR safe mode using the Medtronic MRI SureScan ${ }^{\mathrm{TM}}$ system. At the end of the MRI scan, the device was reprogrammed to the patient's baseline settings with a final report uploaded to the electronic medical record. The protocol resulted in no connectivity or programming errors and no CIED malfunction for the duration of the observation period. Furthermore, the MRI was completed in a safe and timely manner with no requirement of in-person reprogramming. On average, the time saved per scan, which was attributed to the field service representative's travel time, was nearly 30 minutes.

MRI is increasingly becoming the standard imaging modality for diagnostic purposes due to its desirable spatial resolution, tissue characterization, and absence of ionizing radiation. Given these factors, it is predicted that $75 \%$ of patients with a CIED will need an MRI during their lifetime. ${ }^{2}$ With improvement in safety protocols, MRI in patients with CIEDs with either conditional or nonconditional systems is considered safe. ${ }^{1-4}$ However, with improvements in remote monitoring capabilities for CIEDs, there has been a recent desire to expand into remote programming (Table 2). The six primary CIED companies each have a remote monitoring platform. However, Medtronic is the only manufacturer that can offer remote programming. With a growing desire for remote programming capabilities, other CIED manufacturers will likely create similar platforms.

In the background of the COVID-19 era and a desire to reduce provider risk for exposure, several recent studies described using the Medtronic RemoteControl ${ }^{\mathrm{TM}}$ system to program CIEDs upon implant ${ }^{6}$ or during follow-up interactions, such as pre- and post-MR. ${ }^{5,7}$ The programming platform is currently employed only via the Medtronic 2090 programmer with RemoteControl ${ }^{\mathrm{TM}}$ and is an FDA-approved technology that allows for remote operation of the bedside programmer in real-time. ${ }^{5-7}$ The RemoteControl ${ }^{\mathrm{TM}}$ software has embedded security features to authenticate and protect the connection for remote programming and has been successful in delivering successful programming in an expeditious, safe, and reliable manner. ${ }^{5-7}$

Unfortunately, remote programming platforms will present new security risks. CIED monitoring and programming has been threatened by cybersecurity breaches and large scale 'remote hacking' continues to be a primary concern for each of the major CIED manufacturers. ${ }^{8-13}$ With continued software and hardware updates, CIED manufacturers have been able to prevent any significant breaches to remote technologies to date. ${ }^{12,13}$ Remote technologies for CIED have proven to be a safe way to monitor battery status, lead integrity, and device function while allowing for patient safety, economy, and convenience. ${ }^{14}$ In addition, 
they have provided an alert for patient-specific conditions allowing for avoidance of adverse patient events. ${ }^{15}$

At this time, the unidirectional capability of remote home monitoring prevents any significant changes to programmable features of CIEDs, with the exception of arrhythmia detection parameters for implantable cardiac monitors. In contrast, remote programming via the Medtronic RemoteControl ${ }^{\mathrm{TM}}$ system requires both the hardware (Medtronic 2090 programmer) and software (Bomgar ${ }^{\mathrm{TM}}$ and/or CareLink ${ }^{\mathrm{TM}}$ ) to allow for changes to programming parameters, and in this manner provides an additional layer of security. For the foreseeable future, remote programming will continue to require the patient to be in the presence of a dedicated hardware programmer, and rightly so. Any effort to decouple the specialty hardware from the process would carry a risk of malicious hacking that could have catastrophic consequences for a large, vulnerable population. Even if the likelihood of such an event were low, the potential impact would be catastrophic given the number of patients that could be simultaneously affected.

Siddamsetti et al. ${ }^{5}$ present a protocol for remote programming that is likely to be generally adopted with progressive innovations in remote capabilities for CIEDs. Following the significant improvements in remote monitoring over the last two decades ${ }^{15}$, remote programming offers a new phase for CIED companies to deliver expeditious and effective care. The recent COVID-19 pandemic has accelerated a transition toward remote and telehealth capabilities more broadly, with a high level of safety and effectiveness. Although concerns continue to remain regarding cybersecurity features related to the platform, Siddamsetti et al. ${ }^{5}$ have demonstrated that remote programming of CIEDs for MRI can be completed in an expeditious and efficacious manner.

Tables:

Table 1: Definitions for CIED in MRI

\begin{tabular}{ll}
\hline Category & Definition \\
\hline MR safe & Objects that pose no known hazards in all MRI environments \\
MR conditional & Objects that pose no hazards in a specified MRI environment with specified conditions of use \\
MR unsafe & Objects known to pose a risk in all MRI environments \\
MR nonconditional & Objects that have not been declared MR conditional or MR safe \\
\hline
\end{tabular}

$\mathrm{MR}=$ magnetic resonance; modified from Muthalaly et al. ${ }^{2}$

Table 2: Remote Monitoring and Remote Programming Capabilities for Major CIED Manufacturers

\begin{tabular}{lll}
\hline CIED Company & Remote Monitoring Platform & Remote Programming Platform \\
\hline Abbott (Sylmar, CA, USA) & Merlin.Net $^{\mathrm{TM}}$ & - \\
Biotronik (Berlin, Germany) & Home Monitoring $^{\mathrm{TM}}$ & - \\
Boston Scientific (Marlborough, MA, USA) & Latitude $^{\mathrm{TM}}$ & - \\
LivaNova/MicroPort (Paris, France) & Smartview $^{\mathrm{TM}}$ & - \\
Medico (Padova, Italy) & Ermes $^{\mathrm{TM}}$ & - \\
Medtronic (Minneapolis, MN, USA) & Carelink $^{\mathrm{TM}}$ & RemoteControl $^{\mathrm{TM}}$ \\
\hline
\end{tabular}

References:

1. Yang E, Suzuki M, Nazarian S, Halperin HR. Magnetic resonance imaging safety in patients with cardiac implantable electronic devices. Trends Cardiovasc Med . 2021; S1050-1738(21)00085-2.

2. Muthalaly RG, Nerlekar N, Ge Y, Kwong RY, et al. MRI in patients with cardiac implantable electronic devices. Radiology . 2018; 289:281-292.

3. Indik JH, Gimbel JR, Abe H, Alkmim-Teixeira R, et al. 2017 HRS expert consensus statement on magnetic resonance imaging and radiation exposure in patients with cardiovascular implantable electronic 
devices. Heart Rhythm . 2017; 14(7):e97-e153.

4. Paterson DI, White JA, Butler CR, Connelly KA, et al. 2021 Update on Safety of Magnetic Resonance Imaging: Joint statement from Canadian cardiovascular society/Canadian society for cardiovascular magnetic resonance/Canadian heart rhythm society. Can J Cardiol . 2021; 37(6):835-847.

5. Siddamsetti S, Shinn A, Gautam S. Remote programming of cardiac implantable electronic devices: A novel approach to program cardiac devices for magnetic resonance imaging. J Cardiovasc Electrophysiol . 2022; doi

6. Okabe TA, Augostini RA, Hummel JD. Remote programming of cardiac inmplantable electronic devices: Clinical implications in the era of COVID-19. EP Lab Digest. January 2021; 21(1).

7. Kloosterman EM, Rosenbaum M, La Straza B, Wilcox J, et al. Remote control of cardiac implantable electronic devices: Exploring the new frontier-first clinical application of real-time remote-control management of cardiac devices before and after magnetic resonance imaging. J Innov Card Rhythm Manag . 2019; 10(1):3477-3484.

8. Baranchuk A, Refaat MM, Patton KK, Chung MK, et al. Cybersecurity for cardiac implantable electronic devices: What should you know? J Am Coll Cardiol . 2018; 71(11):1284-1288.

9. Alexander B, Haseeb S, Baranchuk A. Are implanted electronic devices hackable? Trends Cardiovasc Med. 2019; 29(8):476-480.

10. Slotwiner DJ, Hoyme KP, Shields S. Cybersecurity of cardiovascular implantable electronic devices and remote programming. Card Electrophysiol Clin . 2021; 13(3):499-508.

11. Tomaiko E, Zawaneh MS. Cybersecurity threats to cardiac implantable devices: Rooms for improvement. Curr Opin Cardiol . 2021; 36(1):1-4.

12. Patel B, Makaryus AN. Cardiac implantable electronic devices and cybersecurity. Expert Rev Med Devices . 2021; 18(sup1):69-77.

13. Das S, Siroky GP, Lee S, Mehta D, et al. Cybersecurity: The need for data and patient safety with cardiac implantable electronic devices. Heart Rhythm . 2021; 18(3):473-481.

14. Lappegard KT, Moe F. Remote monitoring of CIEDs - For safety, economy and Convenience? Int $J$ Environ Res Public Health . 2022; 19:312.

15. Ploux S, Varma N, Strick M, Lazarus A, et al. Optimizing implantable cardioverter-defibrillator remote monitoring: A practical guide.JACC Clin Electrophysiol . 2017; 3(4):315-328. 